\title{
CONTROL OF VIBRATIONAL FIELD IN A VIBRATION UNIT: INFLUENCE OF DRIVE DYNAMICS
}

\author{
Olga P. Tomchina \\ Saint Petersburg State University of Architecture and Civil Engineering (SPSUACE), \\ 2-nd Krasnoarmeiskaya St. 4, 190005, St. Petersburg, Russian Federation \\ otomchina@mail.ru
}

Article history:

Received 23.11.2019, Accepted 27.12.2019

\begin{abstract}
In the paper the problem of feedback control of vibrational fields in a vibration unit is analyzed taking into account the influence of the drive dynamics in the case when the control algorithms are designed when the drive dynamics are neglected. The performance of the closed loop mechatronic systems is examined by simulation for the model of the two-rotor vibration unit SV-2M. Comparison of simulation results for two cases (drive dynamics are neglected or taken into account) is performed.
\end{abstract}

\section{Introduction}

A number of interesting physical phenomena can be found if some engineering methodologies are applied to study physical systems with controllers in the closed feedback loop. For example, an interesting information can be obtained if an engineering system is analyzed taking into account the influence of the drive dynamics in the case when the control algorithms are designed when the drive dynamics are neglected.

In this paper the problem of feedback control of vibrational fields in a vibration unit (VU) is considered. In [Tomchina, 2018] this problem was formulated and studied when the drive dynamics are neglected. The contribution of this paper is the analysis of the case when the drive dynamics are taken into account and comparison of simulation results for two cases (drive dynamics are neglected or taken into account).

VU are used in the mining and manufacturing industries for the purpose of transporting and processing of materials or products: grinding, screening, mixing, compacting, etc. The most effective equipment for vibrational transportation and separation of bulk materials by fractions are vibrating screens that have as their working body a moving screening surface (platform) [Blekhman et al., 2001; Firsova, 2002; Firsova, 2001]. The VU is usually equipped with electromechanical vibration actuators made on the basis of unbalanced rotors (debal- ances) driven by electric drives.

Since different points of a vibrating body oscillate along different trajectories, one may speak about vibrational field. A systematic approach to analysis and synthesis of the vibrational fields for vibrating units was proposed by I.I.Blekhman with coauthors in 2001-2003 [Blekhman et al., 2001; Firsova, 2002; Firsova, 2001] based on the approach of the vibrational mechanics [Blekhman, 2000]. In [Blekhman et al., 2001; Firsova, 2002; Firsova, 2001] the construction of a vibration field for a VU with two actuators is studied. It is shown that it is possible to obtain a more diverse picture of the fields of trajectories in comparison with one-rotor installations. The problem of synthesis and analysis of vibration fields is considered and universal field diagrams are constructed in [Blekhman and Vaisberg, 2011]. It is shown that the vibration field of the two-rotor vibration system depends on the coordinates of the points of fastening of the rotors, the mass of the debalances and the steady phase difference of the debalances provided they are in stable synchronous rotation mode. In [Zhang et al., 2012] is shown that using three actuators may further increase efficiency of the vibration transportation.

Using control algorithms provides additional possibilities for stabilization of synchronous modes of rotation actuators [Andrievskii et al., 2001]. It allows one to stabilize the rotation of unbalanced rotors with a given steady phase difference, since this mode obviously introduces asymmetry into the movement of the platform. An efficient way to implement such a control is the creation of mechatronic units based on the principles of vibration control by feedback. In [Tomchina, 2018] the problem of feedback control of vibrational fields was formulated and studied when the drive dynamics are neglected, In [Andrievsky et al., 2019] the limit possibilities for control of vibrational fields were studied and alternative algorithms of phase shift control were analyzed.

In this paper the influence of the drive dynamics on 




Figure 1. Vibration unit SV-2.

control of vibration fields is examined. Simulation results for the case when the drive dynamics are taken into account and comparison of simulation results for two cases (drive dynamics are neglected or taken into account) are presented. The parameters of the unit model correspond to experimental vibration set-up SV-2 designed in IPME, Saint Petersburg in 1997-2000, see [Andrievskii et al., 2001; Blekhman et al., 1999]. Appearance of SV-2 is shown in Fig. 1. The mathematical model of the unit and the control algorithm are described in Section 2, while the simulation results are presented and analyzed in Section 3.

\section{Model of Two-rotor Vibration Unit Taking into Account the Drive Dynamics}

In this section the mathematical model of the vibration unit and the control algorithm are described following [Tomchina, 2018]. Besides, the drive dynamics model is given, following [Tomchina et al., 2015].

It is a mechatronic (controlled) version of the previous designs developed in Mekhanobr-technika corporation under lead of Prof. Ilya Blekhman. The nomenclature of the variables are presented in Fig. 2, following [Andrievskii et al., 2001].

$M_{r i}(t)=m \varrho g \cos \left(\varphi+\varphi_{i}\right) ; \tilde{M}_{s i}$ is the torque, caused by the influence of the supporting body:

$$
\begin{array}{r}
\tilde{M}_{s i}=-\ddot{x}_{c} m \varrho \sin \left(\varphi+\varphi_{i}\right)+\ddot{y}_{c} m \varrho \cos \left(\varphi+\varphi_{i}\right)+ \\
\ddot{\varphi}\left(J_{i}+(-1)^{i} r m \varrho \cos \varphi_{i}\right)+(-1)^{i} \dot{\varphi}^{2} r m \varrho \sin \varphi_{i},
\end{array}
$$

$\varphi, \varphi_{1}, \varphi_{2}$ are angle of the supporting body and rotation angles of the rotors, respectively, measured from the horizontal position, $x_{c}, y_{c}$ are the horizontal and vertical displacement of the supporting body from the equilibrium position, $m_{i}=m, i=1,2$ and $m_{n}$ are the masses of the rotors and supporting body, $J_{1}, J_{2}$ are the inertia moments of the rotors, $\varrho_{1}=\varrho, i=1,2$ are the rotor eccentricities, $c_{01}, c_{02}$ are the horizontal and vertical spring stiffness, $g$ is the gravity acceleration, $m_{0}$ is the total mass of the unit, $m_{0}=2 m+m_{n}, \beta$ is the damping coefficient, $k_{c}$ is the friction coefficient in the bearings, $M_{i}$ are the motor torques (controlling variables). It is assumed that rotor shafts are orthogonal to the motion of the support.

The structure of the system including electric drives, representing the elastic links of drive shafts with unbalanced rotors has the form presented in Fig. 3, where $M_{i}$ is the electromechanical torque of a motor; $\omega_{i}$ is the angular velocity of a motor; $M_{r i}=k_{r} \cdot \dot{\varphi}_{i}$ is resistance torque of a rotor, caused by resilient friction, $M_{r i}$ is the unbalanced rotor's own torque.

To convert the scheme into the state space equations, assume that the whole system dynamics may be considered in the vertical plane. Then the equations of dynamics have the following form [Tomchina et al., 2015]:

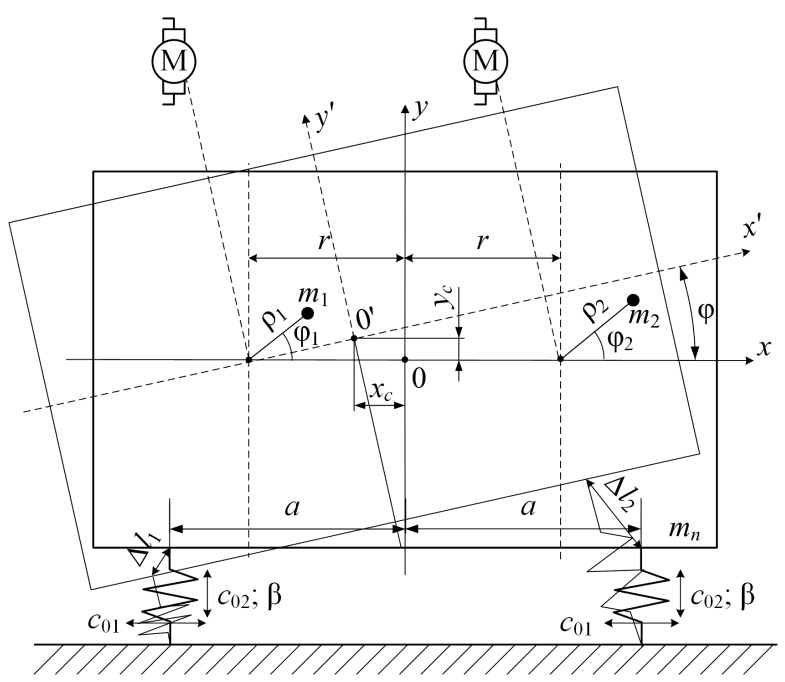

Figure 2. Schematics of two-rotor vibration unit with DC motors. 


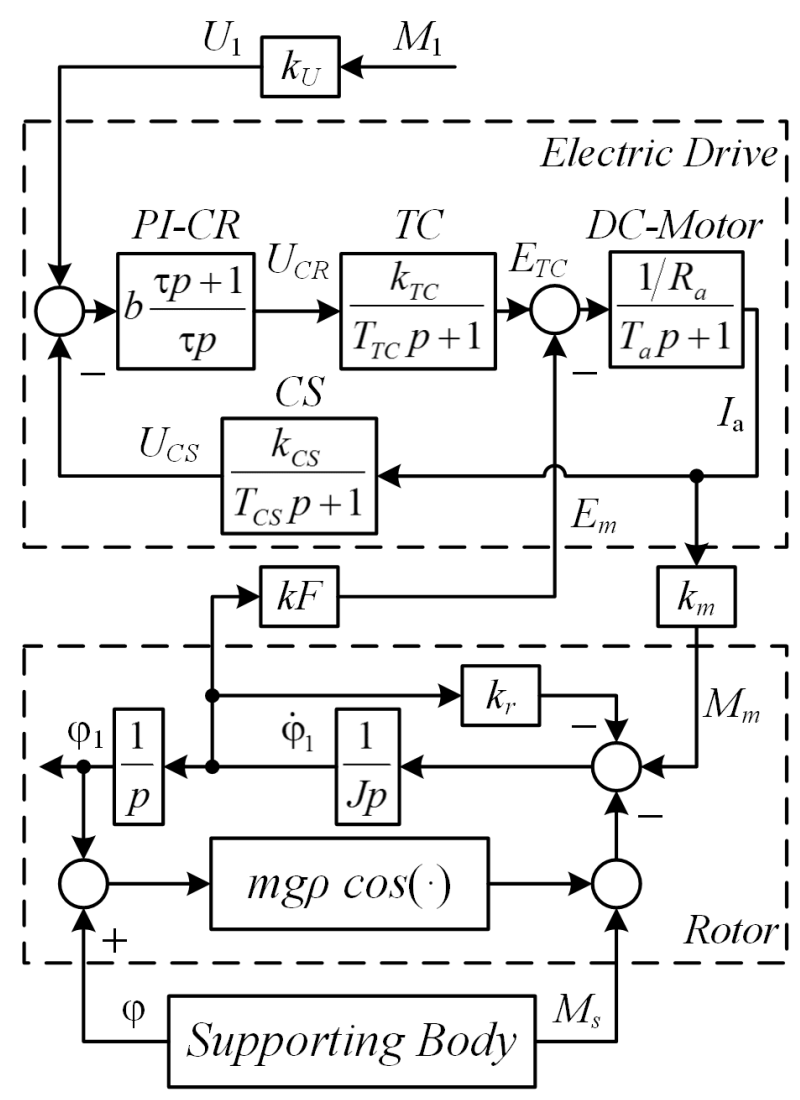

Figure 3. Block diagram of the vibration unit with the drive dynamics.

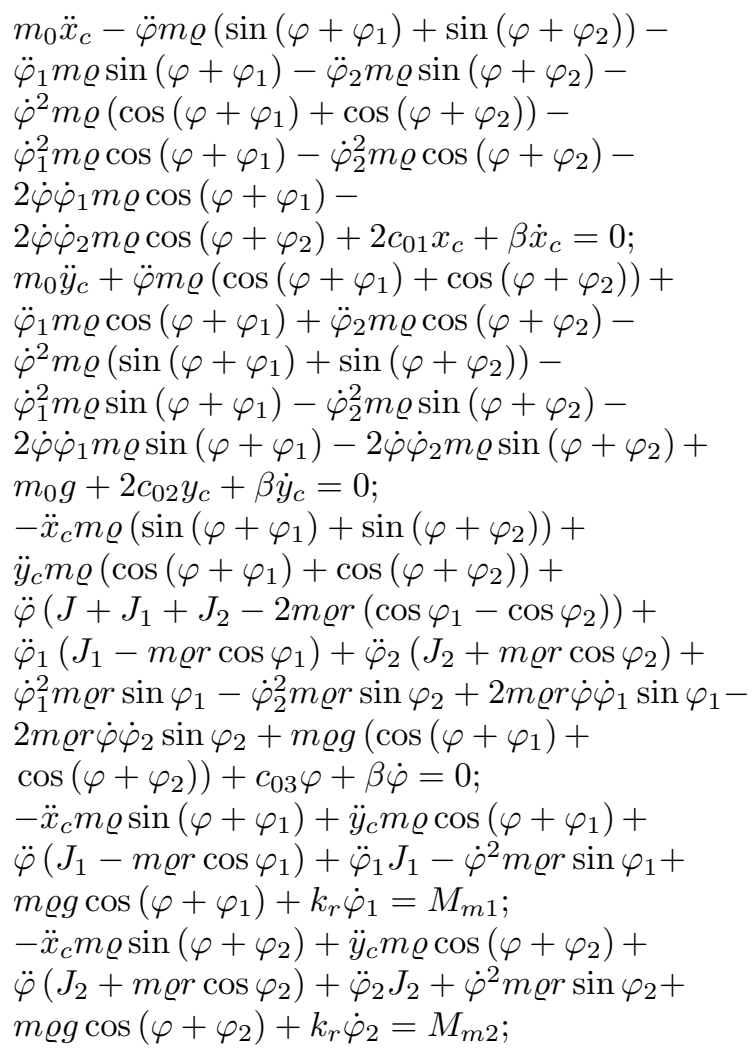

Unbalanced rotor structural diagram taking into account the drive dynamics is shown in Fig. 3. $M_{1}$ is the first drive control torque, the second drive scheme is similar. The main blocks of the system are electric drive, unbalanced rotor, supporting body.

When taking into account the dynamics of the drive in the simulation process the control torque $M_{m 1}$ arriving at the input of the "unbalanced rotor" is formed in accordance with the structural diagram of the "electric drive". Since the laboratory setup SV-2 used the DC motors, the electric drive structure is selected as the traditional single-circuit system with current loop and proportionalintegral (PI) current controller $W_{C R}(p)=b(\tau p+1) / \tau p$ is configured to optimum modulo; $b, \tau$ are dynamic gain and time constant of the regulator. The following notation is used here: $\mathrm{CR}$ is the current regulator; $\mathrm{TC}$ is the power (thyristor type) converter; CS is the current sensor; $I_{a}$ is the armature current; $E_{T C}$ and $E_{m}$ are converter and motor EMFs; $k_{T C}$ and $k_{C S}$ are converter and current feedback gains; $k F$ is the motor torque (EMF) coefficient; $T_{T C}$ and $T_{C S}$ are converter and current sensor time constants; $T_{a}$ is the armature time constant; $R_{a}$ is the armature circuit resistance; $U_{C R}$ and $U_{C S}$ are current controller and current sensor output voltages; $U_{1}$ is the voltage corresponding to the calculated torque $M_{1}$, obtained in accordance with the equations (8), $k_{m}=k F$. The "unbalanced rotor" structural diagram prepared in accordance with the fourth equation of system (2). In accordance with the structure in Fig. 3 each motor torque $M_{m i}$ determined by the value $M_{i}$, calculated in the algorithm and obeys a system of differential and algebraic equations (index " $i$ " is omitted for simplicity):

$$
\begin{array}{r}
\dot{I}_{a}=\frac{1}{T_{a}}\left(-I_{a}+\frac{1}{R_{a}}\left(E_{T C}-E_{m}\right)\right), \\
\dot{E}_{T C}=\frac{1}{T_{T C}}\left(-E_{T C}+k_{T C} U_{C R}\right), \\
\dot{U}_{C S}-\frac{1}{T_{C S}}\left(-U_{C S}+k_{C S} I_{a}\right), \\
\dot{U}_{C S 1}=\frac{b}{\tau}\left(U_{1}-U_{C S}\right), \\
U_{C S}=b\left(U_{1}-U_{C S}\right)+U_{C S 1}, \\
E_{m}=k F \dot{\varphi}, \quad M_{m}=k_{M} I_{a}
\end{array}
$$

\section{Integral-differential Speed-gradient Control Al- gorithms for Multiple Synchronization of Two- rotor Vibration Unit}

Frequency synchronization is defined as an exact coincidence of angular velocities of the unbalanced rotors $\omega_{s}=\omega_{r} ; s, r=1, \ldots, k$. [Blekhman, 2000]. For practice approximate synchronization conditions are more appropriate [Tomchina et al., 2015]:

$$
\left|\omega_{s}-\omega_{r}\right| \leq \varepsilon
$$



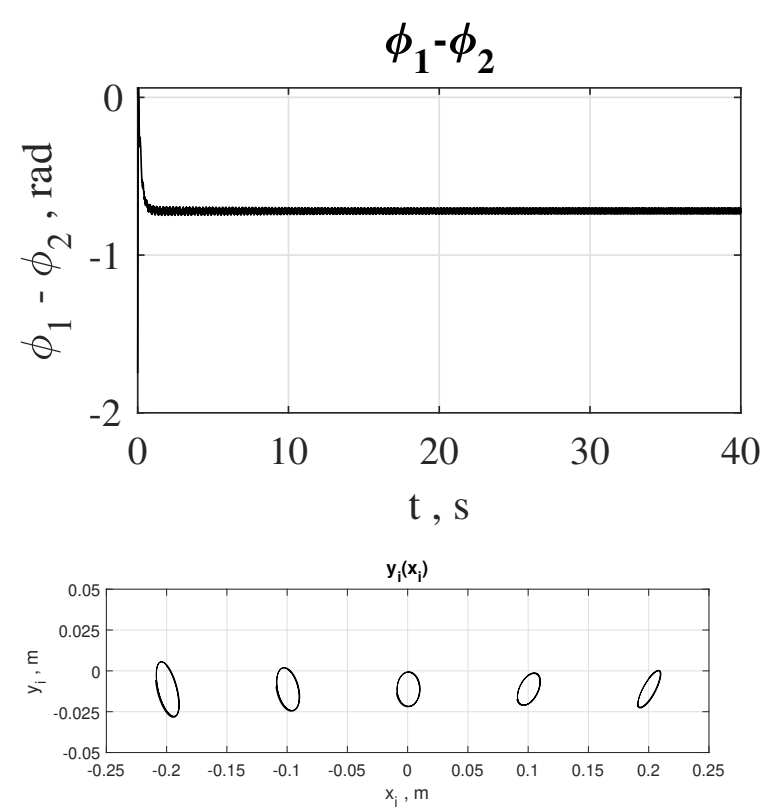

Figure 4. The simulation results for the dynamics of VU neglecting the dynamics of the electric drives $\left(\Delta \varphi_{a d j}=4 \mathrm{rad}, H^{*}=500 \mathrm{~J}\right.$, $\Delta \varphi=-0.74 \mathrm{rad})$

where $\varepsilon>0$ can be chosen numerically as $\varepsilon=0,05 \omega^{*}$, with a given accuracy, similar to conventional transient process measurement. However the ratio (3) may be not sufficient for synchronization, since its fulfillment does not prevent the accumulation of a phase synchronization reduced error (reduced phase shift). That is why there is a need to impose additional requirements on the system phases. To this end the notion of approximate phase synchronization is formulated as follows [Tomchina et al., 2015]:

$$
\left|\varphi_{s}-\varphi_{r}-L_{s r}\right|<\varepsilon ; \quad s, r=1, \ldots, k .
$$

Equations (3) and (4) should hold for some $\varepsilon>0$, and some real $L_{s r}$.

To provide a synchronous rotation mode of unbalanced rotors for system (1), it is suggested to use speedgradient method with an objective functional in the following form:

$$
Q(z)=\left\{0.5(1-\alpha)\left(H-H^{*}\right)^{2}+\alpha\left(\dot{\varphi}_{1} \pm \dot{\varphi}_{2}\right)^{2}\right\},
$$

where $0<\alpha<1$ is weight coefficient; $H$ is total mechanical energy of a system (1), $H^{*}$ is the desired value of $H$. The speed-gradient algorithm in the finite form with the objective functional (5) is as follows [Tomchina, 2018]:

$$
\begin{array}{r}
M_{1}=-\gamma_{1}\left\{(1-\alpha)\left(H-H^{*}\right) \dot{\varphi}_{1}+\right. \\
\left.\frac{\alpha}{J_{1} n_{1}}\left(\frac{\dot{\varphi}_{1}}{n_{1}} \pm \frac{\dot{\varphi}_{2}}{n_{2}}\right)\right\} ; \\
M_{2}=-\gamma_{2}\left\{(1-\alpha)\left(H-H^{*}\right) \dot{\varphi}_{2} \pm\right. \\
\left.\frac{\alpha}{J_{2} n_{2}}\left(\frac{\dot{\varphi}_{1}}{n_{1}} \pm \frac{\dot{\varphi}_{2}}{n_{2}}\right)\right\} ;
\end{array}
$$

For our purpose the proportional-integral (PI-) control algorithm is better suited

$$
\begin{aligned}
M_{1}= & -\gamma_{1}\left\{( 1 - \alpha ) \left[\left(H-H^{*}\right) \dot{\varphi}_{1}+\right.\right. \\
& \left.\int\left(H-H^{*}\right) \dot{\varphi}_{1} d t\right]+\frac{\alpha}{J_{1} n_{1}}\left(\frac{\dot{\varphi}_{1}}{n_{1}} \pm \frac{\dot{\varphi}_{2}}{n_{2}}\right)+ \\
& \left.\frac{\alpha}{J_{1} n_{1}}\left(\frac{\varphi_{1}}{n_{1}} \pm \frac{\varphi_{2}}{n_{2}}+C_{1}\right)\right\} ; \\
M_{2}= & -\gamma_{2}\left\{( 1 - \alpha ) \left[\left(H-H^{*}\right) \dot{\varphi}_{2}+\right.\right. \\
& \left.\int\left(H-H^{*}\right) \dot{\varphi}_{2} d t\right] \pm \frac{\alpha}{J_{2} n_{2}}\left(\frac{\dot{\varphi}_{1}}{n_{1}} \pm \frac{\dot{\varphi}_{2}}{n_{2}}\right) \pm \\
& \left.\frac{\alpha}{J_{2} n_{2}}\left(\frac{\varphi_{1}}{n_{1}} \pm \frac{\varphi_{2}}{n_{2}}+C_{2}\right)\right\} ;
\end{aligned}
$$

where $C_{1}, C_{2}$ are some constant phase shifts. To simplify the implementation of PI-algorithm, the integrals in the first terms in right hand parts of (7) may be omitted:

$$
\begin{aligned}
M_{1}= & -\gamma_{1}\left\{(1-\alpha)\left(H-H^{*}\right) \dot{\varphi}_{1}+\right. \\
& \left.\frac{\alpha}{J_{1} n_{1}}\left(\frac{\dot{\varphi}_{1}}{n_{1}} \pm \frac{\dot{\varphi}_{2}}{n_{2}}\right)+\frac{\alpha}{J_{1} n_{1}}\left(\frac{\varphi_{1}}{n_{1}} \pm \frac{\varphi_{2}}{n_{2}}+\Delta \varphi_{1}^{*}\right)\right\} ; \\
M_{2}= & -\gamma_{2}\left\{(1-\alpha)\left(H-H^{*}\right) \dot{\varphi}_{2} \pm\right. \\
& \left.\frac{\alpha}{J_{2} n_{2}}\left(\frac{\dot{\varphi}_{1}}{n_{1}} \pm \frac{\dot{\varphi}_{2}}{n_{2}}\right) \pm \frac{\alpha}{J_{2} n_{2}}\left(\frac{\varphi_{1}}{n_{1}} \pm \frac{\varphi_{2}}{n_{2}}+\Delta \varphi_{2}^{*}\right)\right\} .
\end{aligned}
$$

The accuracy of such a simplification is reasonable after the transient process, i.e. in the steady-state mode. The choice of the values $\Delta \varphi_{i}^{*}$ in the term $\left(\varphi_{1} / n_{1} \pm \varphi_{2} / n_{2}+\right.$ $\left.\Delta \varphi_{i}^{*}\right)$, allows one to specify the value of the reduced phase shift $\Delta \varphi(\infty)=\varphi_{1}-\varphi_{2}$. In this paper the choice $\Delta \varphi_{2}^{*}=0$ is proposed.

\section{Computer Simulation Results}

In this section, a comparative study of vibration fields is carried out for two cases: A) for the model of the dynamics of the mechanical part of a two-rotor VU without taking into account the dynamics of the electric drive system (equation (1) and B) for the model taking into account the dynamics of the drives (1), (2). For both cases we also examine the influence of the restrictions imposed on the controlling torques.

\subsection{The study of the Vibration Field of a Two-rotor VU without Taking into Account Restrictions on Control Torques}

As indicated earlier, the conclusion that, for fixed coordinates of attachment points and for given mass inertia parameters of vibration exciters, the type of trajectories of the vibration field determined by the steady-state phase shift of the rotors was theoretically justified for a model that takes into account the dynamics of only the mechanical part of the vibration unit. Therefore, at the first stage the vibration fields obtained at the same steady-state phase shift of the rotors were compared for the model of the mechanical part of the vibration unit (1) and the model taking into account the drive (1), (2) by means of simulation.

The results of this study are presented in Fig. 4 and Fig. 5 where a) is the graph of the phase difference of the 
Table 1.

\begin{tabular}{|c|c|c|c|}
\hline \multirow{2}{*}{$H^{*}, \mathrm{~J}$} & \multicolumn{3}{|c|}{$\Delta \varphi \mathrm{vs} H^{*}$ and $\Delta \varphi_{\text {adj }}, \mathrm{rad}$} \\
\cline { 2 - 4 }$\Delta \varphi_{\text {adj }}, \mathrm{rad}$ & 75 & 300 & 500 \\
\hline-4 & 2,1 & 1,115 & 0,79 \\
\hline-3 & 1,55 & 0,795 & 0,575 \\
\hline-2 & 1,025 & 0,52 & 0,39 \\
\hline-1 & 0,52 & 0,265 & 0,195 \\
\hline 0 & 0,03 & 0,02 & 0,015 \\
\hline 1 & $-0,45$ & $-0,225$ & $-0,165$ \\
\hline 2 & $-0,92$ & $-0,475$ & $-0,35$ \\
\hline 3 & $-1,39$ & $-0,74$ & $-0,54$ \\
\hline 4 & $-1,85$ & $-1,035$ & $-0,745$ \\
\hline
\end{tabular}
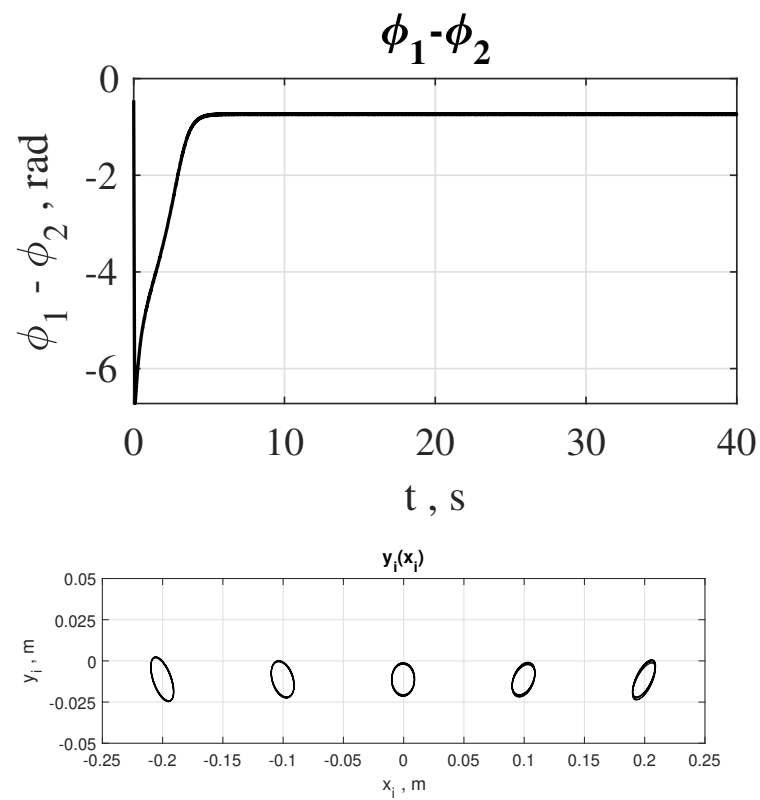

Figure 5. The simulation results for the dynamics of VU neglecting the dynamics of the electric drives $\left(\Delta \varphi_{a d j}=3.7 \mathrm{rad}^{*}=350 \mathrm{~J}\right.$, $\Delta \varphi=-0.74 \mathrm{rad})$

rotors, $\Delta \varphi=\varphi_{1}-\varphi_{2}$, b) are trajectories of movement of 5 points of a platform of the VU. In this section, the parameter of the control algorithm (8) $\Delta \varphi_{1}^{*}$ is denoted by $\Delta \varphi_{1}^{*}=\Delta \varphi_{a d j}$, the parameter $\Delta \varphi_{2}^{*}=0$.

As is seen from the figures, in spite of the difference between the design parameters of the algorithm $\Delta \varphi_{\text {adj }}$ and $H^{*}$ for the same steady-state phase shift $(\Delta \varphi=$ $-0.74 \mathrm{rad}$ ), the slope angles of the main axes of the elliptic trajectories for all points of the platform are almost identical in both cases, which indicates identity of vibration fields. However, as it turned out during the simulation, in order to obtain the same value of the steady-state phase shift in the case of the mechanical part model (1) and for the model taking into account the drive (1), (2) in the control algorithm (8) different values should be specified. For the same $\Delta \varphi_{a d j}$ values in the control algorithm (8), the steady-state values for the systems in question are different. (Fig. 6)

In Fig. 7 and 8 the nomograms which determine the dependence of the steady-state value of the limit phase difference of the rotors $\Delta \varphi$ on the value of the phase difference $\Delta \varphi_{a d j}$ specified in the synchronization control algorithm (8) are presented. Nomograms were obtained for the vibration stand SV-2 model using computer simulation. Each curve of the nomogram corresponds to a certain set of the rotation speed of the rotors, by which the value of $H^{*}$ is determined. In Fig. 7 the nomograms for system (1), not taking into account the drive dynamics are presented. The results for three different values of the desired energy are presented in Table 1.

The similar nomograms for the model of the VU, taking into account the dynamics of electric drives are given below. We assume that the control torques $M_{i}(t)$ are calculated in accordance with formulas (8) and they are not limited in level. The data obtained during simulation are presented in Table 2. According to the data in Table 2 , nomograms for the desired limit phase shift $\Delta \varphi$ vs. $\Delta \varphi_{a d j}$ in presence of the drive dynamics are shown in (Fig. 8).
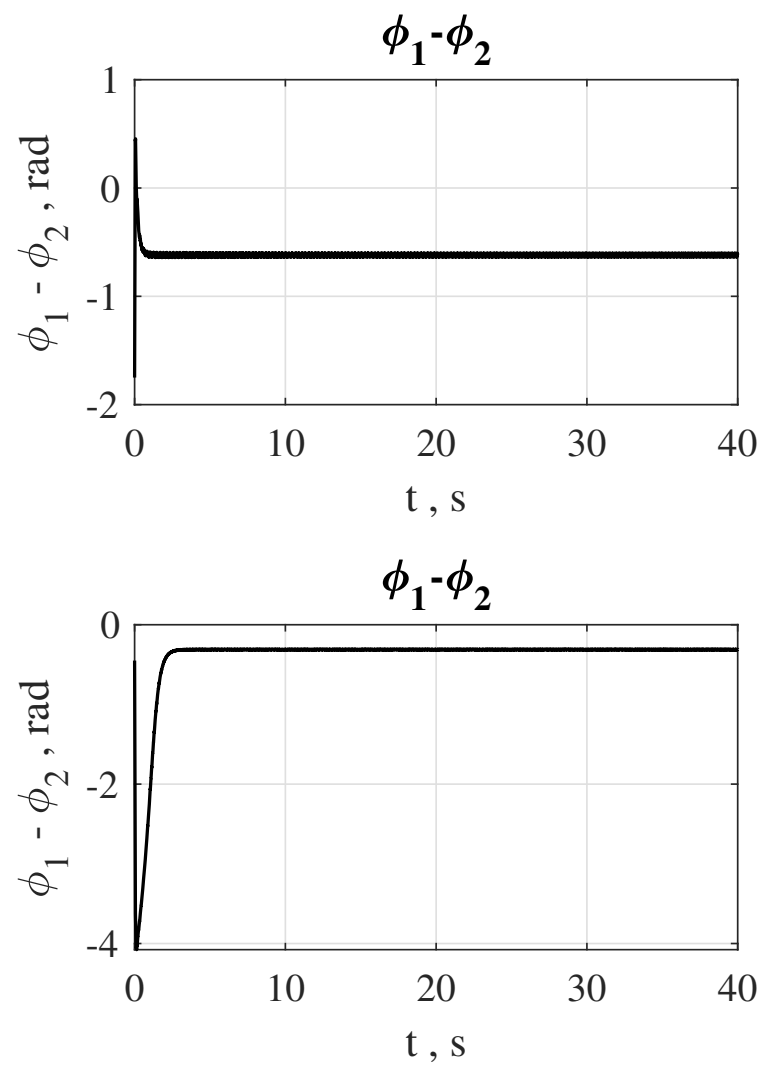

Figure 6. a) $\Delta \varphi=-0.62 \mathrm{rad}$, b) $\Delta \varphi=-0.31 \mathrm{rad}$. Plots of change of $\Delta \varphi$ for $\Delta \varphi_{a d j}=2 \mathrm{rad}, H^{*}=500 \mathrm{~J}$ a) for system (1), b) for system $((1),(2))$ 
Table 2.

\begin{tabular}{|c|c|c|c|}
\hline \multirow{2}{*}{$H^{*}, \mathrm{~J}$} & \multicolumn{3}{|c|}{$\Delta \varphi, \mathrm{rad}$} \\
\cline { 2 - 4 }$\Delta \varphi_{\text {adj }}, \mathrm{rad}$ & $75, \mathrm{~J}$ & $300, \mathrm{~J}$ & $500, \mathrm{~J}$ \\
\hline-5 & 2,525 & 1,415 & 0,925 \\
\hline-4 & 1,995 & 1,01 & 0,698 \\
\hline-3 & 1,46 & 0,705 & 0,5 \\
\hline-2 & 0,94 & 0,45 & 0,324 \\
\hline-1 & 0,455 & 0,214 & 0,155 \\
\hline 0 & $-0,013$ & $-0,013$ & $-0,01$ \\
\hline 1 & $-0,475$ & $-0,238$ & $-0,174$ \\
\hline 2 & $-0,95$ & $-0,47$ & $-0,342$ \\
\hline 3 & $-1,44$ & $-0,73$ & $-0,52$ \\
\hline 4 & $-2,03$ & $-1,025$ & $-0,715$ \\
\hline 5 & $-2,54$ & $-1,425$ & $-0,945$ \\
\hline
\end{tabular}
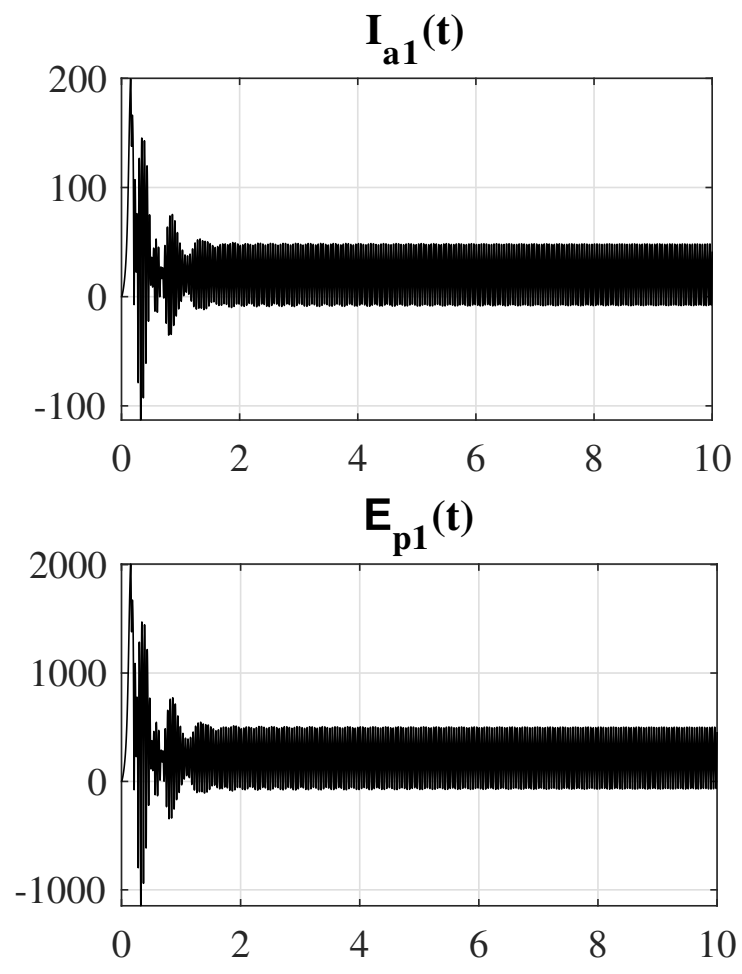

Figure 10. Armature current and EMF TT under constraint $\left|M_{i}\right| \leq$ $L=5 \mathrm{~N} \cdot \mathrm{m}$.



Figure 7. Nomogram representing the dependence of the desired limit phase shift $\Delta \varphi$ on the control algorithm parameter $\Delta \varphi_{a d j}$ for the model (1)



Figure 9. Graphs of changes in armature current and EMF TT without limitation on $M_{i}(t)$.

Figure 8. Nomogram for the desired limit phase shift $\Delta \varphi$ vs. $\Delta \varphi_{a d j}$ in presence of the drive dynamics. 
Table 3 .

\begin{tabular}{|c|c|c|c|c|}
\hline \multirow{2}{*}{$\begin{array}{c}L, \mathrm{~N} \cdot \mathrm{m} \\
\Delta \varphi_{\text {adj }}, \mathrm{rad}\end{array}$} & \multicolumn{4}{|c|}{$\Delta \varphi, \mathrm{rad}$} \\
\cline { 2 - 5 } & 15 & 10 & 5 & 3 \\
\hline-5 & 2,53 & 2,6 & 3,32 & 4,1 \\
\hline-4 & 1,99 & 2 & 1,85 & 2,54 \\
\hline-3 & 1,45 & 1,46 & 1,31 & 0,95 \\
\hline-2 & 0,94 & 0,94 & 0,86 & 0,755 \\
\hline-1 & 0,454 & 0,455 & 0,454 & 0,405 \\
\hline 0 & $-0,013$ & $-0,013$ & $-0,013$ & $-0,013$ \\
\hline 1 & $-0,475$ & $-0,475$ & $-0,475$ & $-0,475$ \\
\hline 2 & $-0,95$ & $-0,95$ & $-0,92$ & $-0,65$ \\
\hline 3 & $-1,45$ & $-1,45$ & $-1,12$ & $-0,89$ \\
\hline 4 & $-2,03$ & $-2,03$ & $-2,67$ & $-3,85$ \\
\hline 5 & $-2,54$ & $-2,6$ & $-3,33$ & $-4,95$ \\
\hline
\end{tabular}

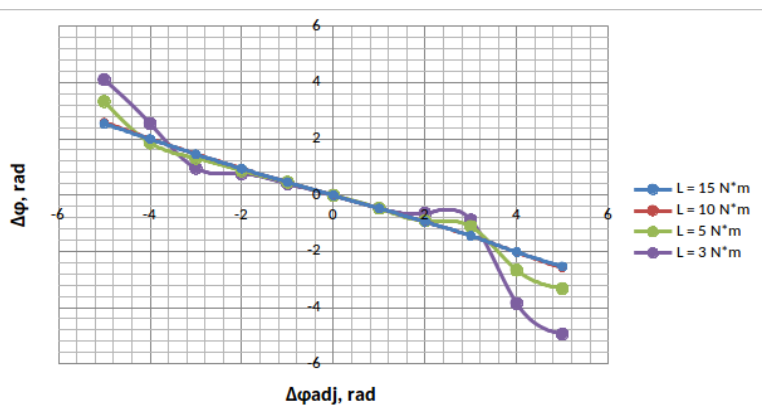

Figure 11. Nomograms of the dependence of the steady-state phase shift on the level of limitation at $H^{*}=75 \mathrm{~J}$.

models taking into account the drive (1), (2) differ. This effect should be taken into account in the practical implementation of control algorithms.

\subsection{Influence of the Constraint for Control Torques on the Steady-state Phase Shift}

The simulation results presented in Fig. 5 and Fig. 8 were obtained under the assumption that the control torques calculated by the formulas of the synchronization algorithm (8) are not limited in magnitude. However, this approach leads to unacceptably large values of the currents and EMF of the thyristor converter in the transition process compared with the normal operating mode. Graphs of changes in the armature current and EMF of the thyristor converter for one of the vibration exciters are shown in Fig. 9.

When limiting the control torques, the current and EMF of the converters have a satisfactory appearance (Fig. 10). Graphs of changes in velocities $\dot{\varphi}_{i}$ and phase differences with limitations are satisfied as well as the requirements for speed and overshoot.

Nomograms of the dependence of the steady-state phase shift on the level of restriction $\left(\left|M_{i}\right| \leq L=\right.$ var; $H^{*}=$ fix) with the parameter of the algorithm (level of the given energy) $H^{*}=75 \mathrm{~J}$ are presented in Fig. 11 .

As is seen from the nomograms of Fig. 8, Fig. 11 and the corresponding data of Table 2 and Table 3 with the magnitude of the constraints on the control torque greater than $10 \mathrm{~N} \cdot \mathrm{m}$, the nomograms obtained for the model taking into account the dynamics of the electric drives coincide with each other (see $L=10 \mathrm{~N} \cdot \mathrm{m}$ and $L=15 \mathrm{~N} \cdot \mathrm{m}$ ) and with nomograms obtained for this model without taking into account restrictions.

Figure 12 shows graphs of changes in the phase difference, rotor speeds, current and trajectories of various points of the platform at $\left|M_{i}\right| \leq L=5 \mathrm{~N} \cdot \mathrm{m}, H^{*}=100$ $\mathrm{J}, \Delta \varphi_{a d j}=3 \mathrm{rad}$. For these parameter values of the control algorithm (8) and this constraint $L$, the steadystate phase shift is $\Delta \varphi=-0.74 \mathrm{rad}$, which corresponds to the simulation results presented in Fig. 5. As can be seen from the graphs, the current with this limitation on the control torque has a slight excess compared with the steady state, and the form of the vibration field is identical to that shown in Fig. 5. Some difference in the areas limited by the trajectories is explained by the difference in the set values of the energy $H^{*}$.

Figure 13 shows the trajectories of the platform points with $\Delta \varphi_{a d j}=-4 \mathrm{rad}, H^{*}=100 \mathrm{~J}$ and $L=5 \mathrm{~N} \cdot \mathrm{m}$. The steady-state phase shift for those parameters of the algorithm is $\Delta \varphi=0.8 \mathrm{rad}$, which leads to a significant difference in the form of the vibration field from the case shown in Fig. 12.

Based on the presented plots we can conclude that when constraints on the motor torque controll of the steady phase shift, using the synchronization algorithm can be implemented by varying the algorithm parameters $H^{*}$ and $\Delta \phi_{a d j}$ within wide limits. However, the nomograms dependencies of $\Delta \phi$ on $\Delta \phi_{a d j}$ Fig.7 depend on the value of the restriction on the motor torques. In addition, with significant restrictions on the motor torques, the dependence of $\Delta \phi$ on $\Delta \phi_{a d j}$ significantly depends on the required operating speeds of the rotors, which are determined by the value of $H^{*}$.

\section{Conclusions}

As shown by the study, the synchronization control algorithm (8), synthesized based on the model (1), provides a stable synchronous mode for the model that takes into account the dynamics of electric drives (1), (2). Moreover, by setting different values of the phase shift $\Delta \phi, \Delta \phi_{a d j}$ in the algorithm (8) for the system (1),(2) it is also possible to obtain different vibration fields corresponding to different steady-state phase shifts $\Delta \phi$. The statement that the vibration field is determined by the value of the steady-state phase shift $\Delta \phi$ which is true for the model with neglected drive dynamics, is also valid for system (1),(2). In addition, for the same value $\Delta \phi$, 

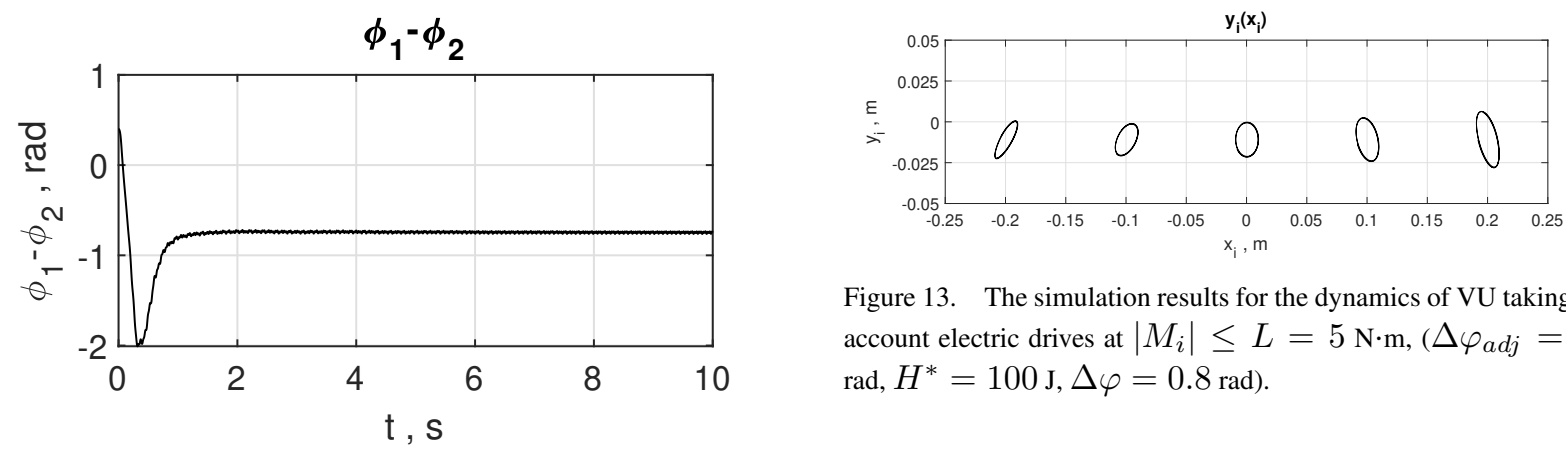

Figure 13. The simulation results for the dynamics of VU taking into account electric drives at $\left|M_{i}\right| \leq L=5 \mathrm{~N} \cdot \mathrm{m},\left(\Delta \varphi_{a d j}=-4\right.$ $\left.\mathrm{rad}, H^{*}=100 \mathrm{~J}, \Delta \varphi=0.8 \mathrm{rad}\right)$.
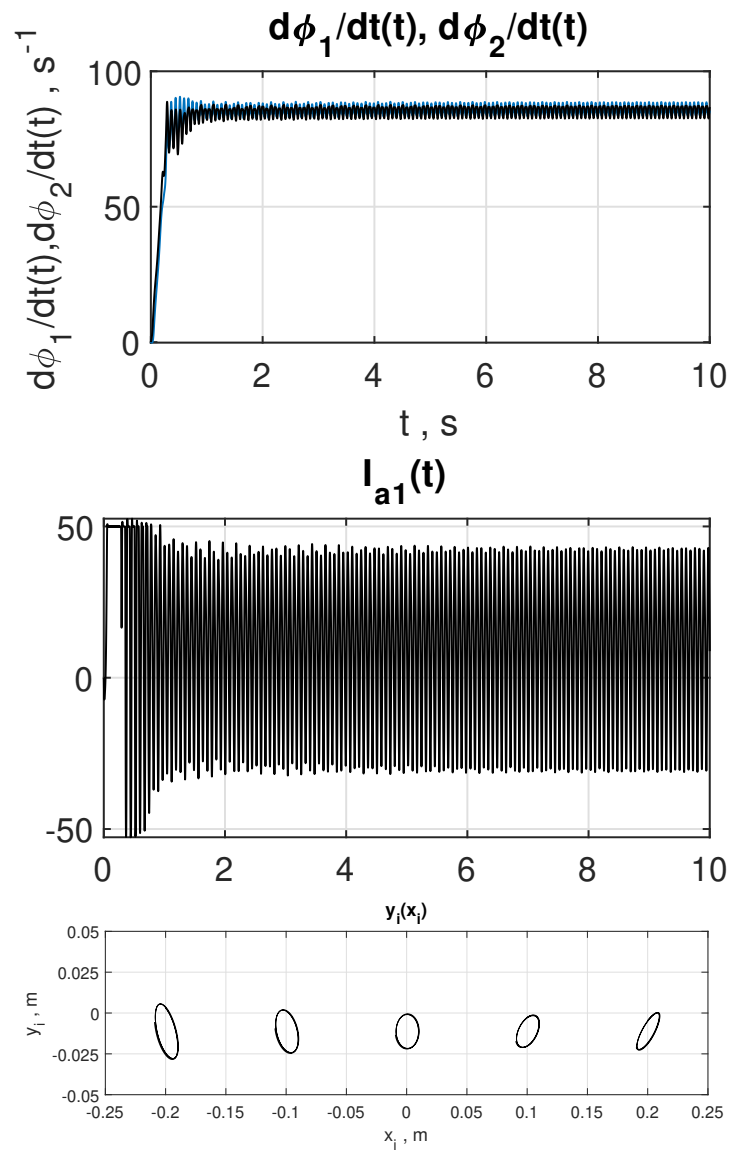

the view of the vibration fields is identical for both models.

It follows from the nomograms (Fig.7,a; Fig.7,b) that for fixed values of the given energy $H^{*}$ the dependences $\Delta \phi$ of $\Delta \phi_{a d j}$ for the system with neglected drive dynamics (1) and taking into account the drive (1), (2) have the form close to linear. However, they differ numerically. Therefore, in practical implementation the dynamics of the drive should be taken into account to provide the desired type of vibration field.

Moreover, the computer study has shown importance of the role played by the magnitude of the maximum controlling torque $L$. Thus, starting from a certain limit value of $\mathrm{L}^{*}$, the graph dependency $\Delta \phi$ from $\Delta \phi_{a d j}$ (Fig.11) at a fixed $H^{*}$ for torques, $\left|M_{i}\right| \leq L^{*}$ practically coincide, while for smaller restrictions $L<L^{*}$ it is necessary to take into account the maximum torque of the motor.

Figure 12. Graphs a) phase difference $\Delta \varphi$, b) rotor speeds, c) current, d) trajectories of various points of the platform for $\left|M_{i}\right| \leq L=$ $5 \mathrm{~N} \cdot \mathrm{m}, H^{*}=100 \mathrm{~J}, \Delta \varphi_{a d j}=3 \mathrm{rad}$. 


\section{References}

Andrievskii, B.R., Blekhman, I.I., Bortsov, Yu.A., Gavrilov, S.V., Konoplev, V.A., Lavrov, B.P., Polyakhov, N.D., Tomchina, O.P., Fradkov, A.L., et al., (2001). Upravlenie mekhatronnymi vibratsionnymi ustanovkami (Control of Mechatronic Vibrational Units); Blekhman, I.I. and Fradkov, A.L., Eds., St. Petersburg: Nauka.

Andrievskii, B.R., Blekhman, I.I., Blekhman, L.I., Boikov, V.I., Vasil'kov, V.B., and Fradkov, A.L. (2016). Education and research mechatronic complex for studying vibration devices and processes. J. Mach. Manuf. Reliab., 45(4), pp. 369-374.

Andrievsky, B., Fradkov, A.L., Tomchina, O., and Boikov, V.I. (2019). Angular Velocity and Phase Shift Control of Mechatronic Vibrational Setup. IFAC Symp. NOLCOS 2019, Vienna, Sept.4-6, pp.1127-1132.

Blekhman, I.I. (2000). Vibrational Mechanics: Nonlinear Dynamic Effects, General Approach, Applications. World Scientific.

Blekhman, I.I., Bortsov, Y.A., Burmistrov, A.A., Fradkov, A.L., Gavrilov, S.V., Kononov, O.A., Lavrov, B.P., Sokolov, P.V., Shestakov, V.M., and Tomchina, O.P. (1999). Computer-controlled vibrational setup for education and research. Proc. of 14th IFAC World Congress, M, pp. 193-197.

Blekhman, I.I., Vaisberg, L.A., and Firsova, A.D. (2001). Evaluation of the field of the trajectories for the body of the vibration machine with two unbalanced vibroactuators. Obogaschenie Rud, No 2, pp. 39-42 (In Russian).
Blekhman, I.I., and Fradkov, A.L. (2004). On general definitions of synchronization. In: Selected topics in vibrational mechanics, Ed. I.I.Blekhman, Singapore: World Scientific, pp. 179-188.

Blekhman, I.I., and Vaisberg, L.A. (2011). Selfsynchronization as a selforganization phenomenon and a basis for development of energy efficient technologies. Proc. of 10th Biennial International Conference on Vibration Problems (ICOVP), Prague, Czech Republic, Sep. 05-08, pp. 365--370.

Firsova A.D. (2001). Investigation of vibration field of a regid body set in motion by two synchronously working vibration exciters Proc. of XXIX Summer school "Advanced Problems in Mechanics" (APM 2001), IPME RAS, pp.231-235.

Firsova A.D. (2002). Stationary oscillations of the operating part of vibration machine with two arbitrary located unbalance vibration exciters Book of Abstracts of the Annual Scientific Conference GAMM 2002, Augsburg, p. 44.

Tomchina, O.P., Gorlatov, D.V., and Tomchin, D.A. (2015). Controlled passage through resonance for two-rotor vibration unit: Influence of drive dynamics. IFAC-PapersOnline, 48(11), pp. 313--318.

Tomchina, O. (2018). Control of vibrational field in a cyber-physical vibration unit. Cybernetics And Physics, 7(3), pp. 144--151.

Zhang, X.-L., Wen, B.C., and Zhao, C.Y. (2012). Synchronization of three homodromy coupled exciters in a non-resonant vibrating system of plane motion. Acta Mechanica Sinica 28(5), pp. 1424-1435. 